Stefaniak Martyna, Pietrzak Zofia, Dzikowski Piotr, Nowicka Emilia, Obel Michal, Piecewicz-Szczęsna Halina. Eslicarbazepine acetate: An efficacy and safety as an antiepileptic drug in clinical trials and open studies. Journal of Education, Health and Sport. 2022;12(2):16-26. eISSN 2391-8306. DOI http://dx.doi.org/10.12775/JEHS.2022.12.02.002

https://apcz.umk.pl/JEHS/article/view/JEHS.2022.12.02.002

https://zenodo.org/record/5946894

The journal has had 40 points in Ministry of Education and Science of Poland parametric evaluation. Annex to the announcement of the Minister of Education and Science of December 1, 2021. No. 32343. Has a Journal's Unique Identifier: 201159. Scientific disciplines assigned: Physical Culture Sciences (Field of Medical sciences and health sciences); Health Sciences (Field of Medical Sciences and Health Sciences).

Punkty Ministerialne z 2019 - aktualny rok 40 punktów. Zalącznik do komunikatu Ministra Edukacji i Nauki z dnia 1 grudnia 2021 r. Lp. 32343. Posiada Unikatowy Identyfikator Czasopisma: 201159. Przypisane dyscypliny naukowe:Nauki o kulturze fizycznej (Dziedzina nauk medycznych i nauk o zdrowiu); Nauki o zdrowiu (Dziedzina nauk medycznych i nauk o zdrowiu).

OThe Authors 2022;
This article is published with open access at Licensee Open Journal Systems of Nicolaus Copernicus University in Torun, Poland

Open Access. This article is distributed under the terms of the Creative Commons Attribution Noncommercial License which permits any noncommercial use, distribution, and reproduction in any medium, provided the original author (s) and source are credited. This is an open access article licensed under the terms of the Creative Commonms Attribution Non commerciail license Share alike.
(http://creativecommons.org/licenses/by-nc-sa/4.0/) which permits unrestricted, non commercial use, distribution and reproduction in any medium, provided the work is properly cited. The authors declare that there is no conflict of interests regarding the publication of this paper.

Received: 02.01.2022. Revised: 17.01.2022. Accepted: 02.02.2022.

\title{
Eslicarbazepine acetate: An efficacy and safety as an antiepileptic drug in clinical trials and open studies
}

Martyna Stefaniak ${ }^{\mathbf{1}}$, martynastefaniakk@gmail.com; https://orcid.org/0000-0002-8036-8379

Zofia Pietrzak ${ }^{1}$, zofer.pietrzak@gmail.com;

https://orcid.org/0000-0001-8469-6225

Piotr Dzikowski ${ }^{1}$, piotrekdzikowski22@gmail.com;

https://orcid.org/0000-0002-6335-9171

Emilia Nowicka ${ }^{1}$, e.nowicka22@gmail.com;

https://orcid.org/0000-0002-2743-1551

Michał Obel'1, michalobel3@gmail.com;

https://orcid.org/0000-0003-1237-8732

Halina Piecewicz-Szczęsna² ${ }^{2}$, halpiec@gmail.com; https://orcid.org/0000-0002-0573-7226

1. Students' Scientific Association at the Chair and Department of Epidemiology, Medical University of Lublin, ul. Radziwiłłowska 11 (Collegium Medicum), 20-080 Lublin, Poland

2. Chair and Department of Epidemiology, Medical University of Lublin, ul. Radziwiłłowska 11 (Collegium Medicum), 20-080 Lublin, Poland

\footnotetext{
Abstract:

Eslicarbazepine acetate (Zebinix ${ }^{\circledR}$, ESL), a voltage-gated sodium channel blocker, is a oncedaily, orally administered anti-seizure medication available in the EU for use as monotherapy in adults with newly diagnosed partial-onset seizures and as adjunctive therapy in adults, adolescents, and children aged $>6$ years with partial-onset seizures. It was approved by the European Medicines Agency and launched onto the European market in 2009.
} 
This study aimed to assess the efficacy and safety of ESL in the treatment of focal-onset seizures. Our study material consisted of publications, which were found in PubMed, Google Scholar, and Embase databases. In order to find the proper publications, the search has been conducted with the use of a combination of keywords like: "eslicarbazepine acetate", "focalonset seizures treatment", "epilepsy treatment", "eslicarbazepine acetate pharmacokinetics". The first step was to find proper publications from the last 10 years. The second step was to carry out an overview of the found publications.

Results of mentioned studies proved that for adults with medically uncontrolled partial-onset seizures, ESL monotherapy is well tolerated and effective over the long term, including the patients who transitioned from CBZ-CR monotherapy. Adjunctive ESL demonstrated a sustained therapeutic effect and was well-tolerated, safe, and efficacious during the treatment of adults with partial-onset seizures. Both $800 \mathrm{mg}$ and $1200 \mathrm{mg}$ once-daily doses were well tolerated. Moreover, significant improvements in depressive symptoms and quality of life domains were observed under long-term treatment with ESL.

Key words: Eslicarbazepine acetate; Focal-onset seizures; Monotherapy; Adjunctive therapy

\section{INTRODUCTION AND PURPOSE}

Eslicarbazepine acetate (Zebinix $\left.{ }^{\circledR}, \mathrm{ESL}\right)$, a voltage-gated sodium channel blocker, is a once-daily, orally administered anti-seizure medication available in the EU for use as monotherapy in adults with newly diagnosed partial-onset (focal-onset) seizures and as adjunctive therapy in adults, adolescents, and children aged $>6$ years with partial-onset seizures [1,2]. It was approved by the European Medicines Agency and launched onto the European market in 2009 [3,4]. Together with carbamazepine (CBZ) and oxcarbazepine, ESL belongs to the dibenzazepine family; ESL has few, but some, drug-drug interactions $[5,6]$. It is a weak enzyme inducer and it inhibits cytochrome P450 2C19, but it affects a smaller assortment of enzymes than carbamazepine [6]. Some patients diagnosed with epilepsy may continue suffering from seizures despite treatment with antiepileptic drugs, either in monotherapy or polytherapy, therefore there remains the need to develop new effective and well-tolerated therapies [7].

This study aimed to assess the efficacy and safety of ESL in the treatment of focalonset seizures. Our study material consisted of publications, which were found in PubMed, Google Scholar, and Embase databases. In order to find the proper publications, the search has been conducted with the use of a combination of keywords like: "eslicarbazepine acetate", "focal-onset seizures treatment", "epilepsy treatment", "eslicarbazepine acetate pharmacokinetics". The first step was to find proper publications from the last 10 years. The second step was to carry out an overview of the found publications. 


\section{RESULTS}

\section{Efficacy and safety of eslicarbazepine acetate monotherapy in adults with partial-onset seizures}

An open-label extension (OLE) study was conducted by Trinka E. et al. to assess the efficacy, safety, and tolerability of ESL monotherapy during long-term treatment. The study was performed in adults completing a phase 3, randomized, double-blind, noninferiority trial, during which they had received monotherapy with either once-daily ESL or twice-daily controlled-release carbamazepine (CBZ-CR) for newly diagnosed focal epilepsy. In the OLE study, all patients received ESL (800-1600 mg/d) for 2 years. The primary efficacy outcome was retention time (from baseline of the OLE study). Secondary efficacy assessments included seizure freedom rate (no seizures during the OLE study) and responder rate ( $\geq 50 \%$ seizure frequency reduction from baseline of double-blind trial). Safety assessments included evaluation of treatment-emergent adverse events (TEAEs).

Of 206 randomized patients, 96 who received ESL in the double-blind trial (ESL/ESL) and 88 who received CBZ-CR in the double-blind trial (CBZ-CR/ESL) were treated with ESL monotherapy ( $89.3 \%$ overall). The treatment retention time was similar between groups, with a low probability of ESL withdrawal overall $(<0.07$ at any time). After 24 months, the probability of ESL withdrawal was $0.0638(95 \%$ confidence interval $[\mathrm{CI}]=0.0292-0.1366)$ in the ESL/ESL group and $0.0472(95 \% \mathrm{CI}=0.0180-0.1210)$ in the CBZ-CR/ESL group. Seizure freedom rates were $90.6 \%$ (ESL/ESL) and $80.7 \%(\mathrm{CBZ}-\mathrm{CR} / \mathrm{ESL} ; \mathrm{P}=.0531)$. Responder rates remained $>80 \%$ in both groups throughout the study. Incidence of serious TEAEs was similar between groups ( $7.3 \%$ vs $5.7 \%$; $0 \%$ vs $1.1 \%$ possibly related), as were the incidences of TEAEs considered at least possibly related to treatment $(17.7 \%$ vs $18.2 \%)$ and TEAEs leading to discontinuation (3.1\% vs $4.5 \%)$. The types of TEAEs were generally consistent with the known safety profile of ESL [8].

Pazdera L. et al. conducted a study to evaluate the influence of prior use of CBZ and other antiepileptic drugs (AEDs) with a putatively similar mechanism of action (inhibition of voltage-gated sodium channels; VGSCs) on seizure outcomes and tolerability when converting to ESL, using data pooled from 2 controlled conversion-to-ESL monotherapy trials.

Adults with treatment-resistant partial-onset seizures were randomized 2:1 to ESL 1600 or $1200 \mathrm{mg}$ once daily. The primary efficacy endpoint was study exit (meeting predefined exit criteria related to worsening seizure control) versus a historical control group. Other endpoints included changes in seizure frequency, responder rate, and tolerability. Endpoints were analyzed for subgroups of patients who received CBZ (or any VGSC inhibitor [VGSCi]) during baseline versus those who received other AEDs.

Of 365 patients in the studies, 332 were evaluable for efficacy. The higher risk of study exit in the subgroups that received CBZ (or any VGSCi) during baseline, versus other AEDs, was not statistically significant (hazard ratios were 1.49 for $+\mathrm{CBZ}$ vs $-\mathrm{CBZ}[\mathrm{P}=.10]$ and 1.27 for +VGSCi vs. -VGSCi $[\mathrm{P}=.33]$ ). Reductions in seizure frequency and responder rates were lower in patients who converted from CBZ or other VGSCi compared with those who converted from other AEDs. There were no notable differences in overall tolerability between subgroups, but the incidence of some adverse events (eg, dizziness, somnolence, nausea) differed between subgroups and/or between treatment periods. 
Baseline use of CBZ or other major putative VGSC inhibitors did not appear to significantly increase the risk of study exit due to worsening seizure control or to increase the frequency of side effects when converting to ESL monotherapy. However, bigger improvements in efficacy may be possible in patients converting to ESL monotherapy from an AED regimen that does not include a VGSCi [9].

Sperling M. et al. carried out post hoc pooled analysis of 2 randomized double-blind studies to assess the efficacy and safety of ESL monotherapy.

These studies included adults with partial-onset seizures medically uncontrolled by 1 or 2 AEDs. Following the baseline period ( 8 weeks), eligible patients were randomized 2:1 to receive ESL $1,600 \mathrm{mg}$ or 1,200 $\mathrm{mg}$ once daily for 18 weeks; the primary endpoint was study exit by meeting predefined exit criteria (signifying worsening seizure control). In each study, treatment was considered effective if the upper $95 \%$ confidence limit for exit rate was lower than the historical control threshold (65.3\%).

Pooled exit rates were as follows: ESL $1,600 \mathrm{mg}=20.6 \%(95 \%$ confidence interval: 15.6\%-26.8\%); ESL 1,200 mg $=30.8 \%(23.0 \%-40.5 \%)$. Use of 2 baseline AEDs or rescue medication, US location, epilepsy duration $\geq 20$ years, and higher maximum baseline seizure frequency were associated with higher exit risks. Median percent reductions in standardized seizure frequency between baseline and the 18-week double-blind period were as follows: ESL $1,600 \mathrm{mg}=43.2 \%$; ESL 1,200 $\mathrm{mg}=35.7 \%$; baseline carbamazepine use was associated with smaller reductions. Safety profiles were similar between ESL doses.

Exit rates for ESL monotherapy (1,600 $\mathrm{mg}$ and 1,200 $\mathrm{mg}$ once daily) were lower than the historical control threshold, irrespective of baseline AED use and region, with no additional safety concerns identified. Clinical factors and location clearly influence treatment responses in conversion-to-monotherapy trials [10].

\section{Efficacy and safety of eslicarbazepine acetate as adjunctive therapy in adults with partial-onset seizures}

This randomized, placebo-controlled, double-blind, parallel-group, phase III study was conducted by Sperling M. et al. at 173 centers in 19 countries, including the United States and Canada to evaluate the efficacy and safety of adjunctive ESL in patients with refractory partial-onset seizures. Eligible patients were aged $\geq 16$ years and had uncontrolled partialonset seizures despite treatment with 1-2 AEDs. After an 8-week baseline period, patients were randomized to once-daily placebo $(\mathrm{n}=226)$, ESL $800 \mathrm{mg}(\mathrm{n}=216)$, or ESL 1,200 mg ( $=211$ ). Following a 2-week titration period, patients received ESL 800 or 1,200 mg once daily for 12 weeks. Seizure data were captured and documented using event-entry or daily entry diaries.

Standardized seizure frequency (SSF) during the maintenance period (primary end point) was reduced with ESL $1,200 \mathrm{mg}(\mathrm{p}=0.004)$, and there was a trend toward improvement with ESL $800 \mathrm{mg}(\mathrm{p}=0.06)$, compared with placebo. When data for titration and maintenance periods were combined, ESL $800 \mathrm{mg}(\mathrm{p}=0.001)$ and 1,200 $\mathrm{mg}(\mathrm{p}<0.001)$ both reduced SSF. There were no statistically significant interactions between treatment response and geographical region $(\mathrm{p}=0.38)$ or diary version $(\mathrm{p}=0.76)$. Responder rate ( $\geq 50 \%$ reduction in SSF) was significantly higher with ESL 1,200 mg $(42.6 \%, p<0.001$ ) but not ESL $800 \mathrm{mg}(30.5 \%, \mathrm{p}=0.07)$ than placebo $(23.1 \%)$. Incidence of TEAEs and TEAEs 
leading to discontinuation increased with ESL dose. The most common TEAEs were dizziness, somnolence, nausea, headache, and diplopia [11].

The aim of the study performed by Costa R. et al. was to evaluate the safety, tolerability, and efficacy of ESL as adjunctive therapy in patients aged $\geq 65$ years with focalonset seizures (FOS). This was an international, multicentre, open-label, non-controlled, single-arm, post-European approval commitment study with flexible doses of ESL between 400 and $1200 \mathrm{mg}$ /day. Seventy-two elderly patients with at least two FOS in the prior 4 weeks, and treated with one or two AEDs, were enrolled. The study consisted of an 8-week baseline, followed by a 26 -week treatment period during which the investigator was allowed to up- or down-titrate the ESL dose, and a 4-week follow-up period. Safety and tolerability were assessed as well as mental sedation, cognitive mental state, and suicidal ideation. Efficacy was assessed based on patient diaries regarding the absolute and relative changes in seizure frequency, change in intellectual impairment, and quality of life.

Overall, 47 (65.3\%) patients experienced 152 TEAEs. The most frequent were dizziness (12.5\%), somnolence (9.7\%), fatigue, convulsion, and hyponatremia ( $8.3 \%$ each). All patients that experienced hyponatremia (6/72) recovered without sequelae. Three patients died during the study (due to cardiac failure, glioblastoma multiforme, and ischaemic stroke, all considered unrelated to ESL). Overall, 16 (22.2\%) patients discontinued prematurely due to TEAEs. The incidences of clinically significant findings were low for vital signs, ECG, physical and neurological examinations. No TEAEs of hypothyroidism were reported; however, 24 (33.3\%) patients presented post-baseline shifts from normal to decreased free T4 levels (not clinically significant). ESL decreased standardized seizure frequency from a mean of 4.8 seizures at baseline to 3.6 seizures at endpoint ( $p>0.05)$; and mean number of days with seizures significantly decreased from 4.1 (baseline) to 2.8 at endpoint $(p=0.0408)$ [12].

The aim of the study conducted by Hufnagel A. et al. was to evaluate the long-term safety, tolerability, and efficacy of once-daily ESL as adjunctive therapy in adults with partial-onset seizures. They performed a one-year OLE study with ESL in patients who completed a randomized, double-blind placebo-controlled trial. Starting dose was $800 \mathrm{mg}$ once daily, for 4 weeks; thereafter, the dose could be individualized within the 400-1,200 mg range. Doses of concomitant antiepileptic drugs were to be kept stable.

Overall, 325 patients were enrolled (intent-to-treat population); 223 (68.6\%) patients completed 1-year of treatment. ESL median dose was $800 \mathrm{mg}$ once daily. Compared to the baseline period of the double-blind study completed prior to this OLE study, median seizure frequency decreased by $32 \%$ in weeks $1-4$, and between $37 \%$ and $39 \%$ thereafter. The responder rate (seizure reduction $\geq 50 \%$ ) was $37 \%$ during weeks $1-4$ and thereafter ranged between $38 \%$ and $42 \%$ per 12 -week interval. The proportion of seizure-free patients per $12-$ week interval ranged between 5\% and 11\%. Improvements from baseline in several Quality of Life in Epilepsy Inventory-31 (QOLIE-31) and Montgomery Asberg Depression Rating Scale (MADRS) scores were observed. Adverse events (AEs) were reported by $83 \%$ of patients. AEs occurring in $\geq 10 \%$ of patients were dizziness, headache, and somnolence. AEs were usually of mild to moderate intensity [13]. 


\section{Social cognition and cognitive functions in patients treated with eslicarbazepine acetate}

Abraira L. et al. conducted a prospective single-center study with patients aged between 18 and 65 years with focal seizures treated with ESL to analyze the impact of treatment with ESL on social cognition and prefrontal cognitive functions in adults with focal epilepsy. The patients were evaluated in their baseline visit and at six months after starting ESL treatment by means of tasks designed for theory of mind, executive and attentional functions, auditory-verbal memory, quality of life, and anxiety and depression.

Forty-one patients were treated with ESL, and 30 completed the follow-up. A significant improvement was observed in the theory of mind tasks. In the analysis stratified by sex, the men showed greater improvement. A cognitive improvement was observed in the Wisconsin Card Sorting Test, Symbol Digit, Backward Digit Span, and Stroop tests. No differences were found in the Quality of Life in Epilepsy-31 Inventory or in the Hospital Anxiety and Depression Scale. These results were independent of the reduction in the number of seizures and the ESL dosage [14].

\section{Effects of eslicarbazepine acetate on cardiac repolarization}

The study conducted by Vaz-Da-Silva M. et al. investigated the effect of ESL on cardiac repolarization in healthy adult volunteers. A randomized, placebo/active-controlled, 4period crossover study was conducted in 67 participants. In 3 periods, participants received once-daily doses of ESL $1200 \mathrm{mg}$, ESL $2400 \mathrm{mg}$, and placebo for 5 days; in 1 period, participants received a placebo on days 1 to 4 and a 400-mg moxifloxacin single dose on day 5. In each period, 24-hour 12-lead Holter monitoring was performed on days -;1 (baseline) and 5. There was no clinically relevant effect of ESL $1200 \mathrm{mg}$ and $2400 \mathrm{mg}$ versus placebo on cardiac depolarization or repolarization as measured by the QRS or QTc intervals, respectively. Mean PR interval increased following ESL $1200 \mathrm{mg}$ and $2400 \mathrm{mg}$, but there was no participant with a PR interval above the upper limit of the normal range $(200 \mathrm{~ms})$. The upper bound of the $95 \%$ confidence interval for the placebo-corrected change from baseline of the individually corrected QT interval (QTcI) following administration of ESL $1200 \mathrm{mg}$ and ESL $2400 \mathrm{mg}$ was $<10 \mathrm{~ms}$ at every time point. Moxifloxacin caused an increase in QTcI above the 10-ms threshold for clinical significance at several time points, demonstrating assay sensitivity [15].

\section{Effect of eslicarbazepine acetate on serum lipids and the pharmacokinetics of statins}

To evaluate the effects of ESL on lipid metabolism and to determine whether reduced statin exposure during ESL therapy has clinical consequences Mintzer S. et al. conducted a post-hoc analysis of pooled data for serum lipids (laboratory values) from three phase III, multicenter, randomized, double-blind, placebo-controlled trials of adjunctive ESL therapy $(400,800$, or $1200 \mathrm{mg}$ once daily) in patients with treatment-refractory partial-onset seizures. Changes from baseline in serum lipid levels were analyzed according to the use of statins and/or enzyme-inducing antiepileptic drugs (EIAEDs) during the baseline period.

In total, 426 and 1021 placebo- and ESL-treated patients, respectively, were included in the analysis. With regard to the changes from baseline in serum concentrations, there were statistically significant differences between the placebo and ESL $1200 \mathrm{mg}$ once a day (QD) groups, for both total cholesterol (TC) and high-density lipoprotein cholesterol (HDL-C), but 
the effect sizes were small $(+4.1 \mathrm{mg} / \mathrm{dL}$ and $+1.8 \mathrm{mg} / \mathrm{dL}$, respectively). A small but significant difference in low-density lipoprotein cholesterol (LDL-C; -5.0 mg/dL) was observed between the ESL $400 \mathrm{mg}$ QD group and the placebo group. In patients not taking a concomitant EIAED, there were no changes with ESL $400 \mathrm{mg}$ QD, but modest and statistically significant increases in cholesterol fractions (TC, LDL-C, and HDL-C) with ESL $800 \mathrm{mg}$ QD $(<6 \mathrm{mg} / \mathrm{dL})$ and ESL $1200 \mathrm{mg}$ QD $(<10 \mathrm{mg} / \mathrm{dL})$. ESL had no consistent effect on lipids in patients taking a concomitant EIAED. In patients taking statins during baseline, there were no clinically relevant changes in serum lipids during use of ESL, although the subgroups were small [16].

Single-center, two-way cross-over, randomized, open-label study in 24 healthy volunteers was performed by Falcao A. et al. to investigate the effect of ESL on the pharmacokinetics of simvastatin (SMV), a known CYP3A4 substrate, in healthy subjects. The volunteers received an oral single dose of SMV 80mg on two occasions (once administered alone and once after treatment with an oral once-daily dose of $800 \mathrm{mg}$ of ESL for 14 days), separated by a wash-out period of 3 weeks or more. The analysis of variance (ANOVA) was used to test for differences between Test (SMV under co-administration with ESL) and Reference (SMV administered alone) treatments for AUC0- $\infty$, AUC0-t, and Cmax of SMV and SMV-acid.

Mean systemic exposure (AUC) measurements for both SMV and SMV- $\beta$ hydroxyacid (SMV-acid) were up to 54\% lower during ESL use. The Test/Reference geometric mean ratios (GMR) $(90 \%$ CI) for the AUC0-t of SMV and SMV-acid were $46 \%$ $(38 \% ; 55 \%)$ and $49 \%(44 \% ; 55 \%)$, respectively. Mean peak concentrations (Cmax) of both SMV and SMV-acid were reduced by $60 \%$ and $41 \%$, respectively, when SMV was administered with ESL [17].

Patients with partial-onset seizures and comorbid cardiovascular disease may concomitantly receive ESL, an antiepileptic drug, and rosuvastatin, an HMG-CoA reductase inhibitor. The study conducted by Gidal et al. evaluated the effect of multiple-dose ESL on the pharmacokinetic (PK) parameters of a single dose of rosuvastatin in healthy subjects.

This was a Phase I, single-center, fixed-sequence, open-label study. Healthy subjects received two treatments, in sequence. Treatment A: a single 40mg oral dose of rosuvastatin on Day 1, followed by a washout period (Days 1-4); treatment B: titration of ESL (400-800mg once daily) on Days 5-18, followed by ESL 1200mg once daily on Days 19-35, with a single dose of rosuvastatin $(40 \mathrm{mg})$ on Day 32. Subjects then entered a 2-week follow-up period. Plasma concentrations of rosuvastatin were quantified for PK analyses. Safety and tolerability were assessed throughout the study.

Thirty-three healthy subjects were enrolled and 30 completed the study. Mean rosuvastatin (standard deviation) $\mathrm{t} 1 / 2$ was similar when rosuvastatin was used concomitantly with ESL and when it was used alone. The geometric least-squares mean ratios $(90 \%$ confidence intervals) of rosuvastatin exposure levels between rosuvastatin used concomitantly with ESL and rosuvastatin used alone were as follows: Cmax, 64.0\% (55.9-73.3\%); AUC(0$\infty), 63.0 \%$ (57.1-69.4\%); and AUC(0-last), 60.9\% (55.2-67.1\%). Concomitant use of ESL and rosuvastatin was generally well tolerated.

Rosuvastatin exposure was 36-39\% lower with steady-state administration of ESL, potentially due to reduced oral bioavailability of rosuvastatin [18]. 


\section{CONCLUSIONS}

1. For adults with medically uncontrolled simple partial seizures, ESL monotherapy is well tolerated and effective over the long term, including the patients who transitioned from CBZ-CR monotherapy.

2. Adjunctive ESL demonstrated a sustained therapeutic effect and was well-tolerated, safe, and efficacious during the treatment of adults with simple partial seizures. Both $800 \mathrm{mg}$ and $1200 \mathrm{mg}$ once-daily doses were well tolerated. Moreover, significant improvements in depressive symptoms and quality of life domains were observed under long-term treatment with ESL.

3. ESL could improve some aspects of the theory of mind in patients with epilepsy, especially in men and independently of the control of seizures, with no changes in quality of life, anxiety, or depression.

4. The administration of both $1200 \mathrm{mg}$ and $2400 \mathrm{mg}$ of ESL did not induce a clinically significant prolongation of the QTcI interval.

5. A significant effect of repeated ESL administration on the pharmacokinetics of SMV and rosuvastatin was observed. If a clinically significant change in lipids is noted, dose adjustment of SMV and rosuvastatin may be required when used concomitantly with ESL.

\footnotetext{
Abbreviations

ESL - eslicarbazepine acetate

$\mathrm{CBZ}$ - carbamazepine

OLE - open-label extension

CBZ-CR - controlled-release carbamazepine

TEAEs - treatment-emergent adverse events

AEDs - antiepileptic drugs

VGSCs - voltage-gated sodium channels

VGSCi - voltage-gated sodium channels inhibitor

SSF - standardized seizure frequency

FOS - focal-onset seizures

QOLIE-31 - Quality of Life in Epilepsy Inventory-31

MADRS - Montgomery Asberg Depression Rating Scale

AEs - adverse events

QTcI - QT interval

EIAEDs - enzyme-inducing antiepileptic drugs

QD - once a day

TC - total cholesterol

HDL-C - high-density lipoprotein cholesterol

LDL-C - low-density lipoprotein cholesterol

SMV - simvastatin

ANOVA - analysis of variance

AUC - mean systemic exposure
} 
SMV-acid - SMV- $\beta$-hydroxyacid

GMR - geometric mean ratios

Cmax - mean peak concentrations

PK - pharmacokinetic

\section{Author contributions}

All the authors made substantial contributions to the conception or design of the work, or the acquisition, analysis, or interpretation of data for the work; and were involved in drafting the work and revising it critically for important intellectual content; and gave final approval for the version to be published; and agree to be accountable for all aspects of the work in ensuring that questions related to the accuracy or integrity of any part of the work are appropriately investigated and resolved.

\section{Financial \& competing interests disclosure}

This study was not sponsored and funded.

\section{Ethical conduct of research}

No ethical approval was required for this research.

\section{Data sharing statement}

Any additional datasets that are not provided as part of the manuscript or as supplementary materials are available from the corresponding author on reasonable request.

\section{LIST OF REFERENCES}

1. Heo YA. Eslicarbazepine Acetate: A Review in Focal-Onset Seizures. CNS Drugs. 2020 Sep;34(9):989-1000. doi: 10.1007/s40263-020-00751-3. PMID: 32737793.

2. Shorvon SD, Trinka E, Steinhoff BJ, Holtkamp M, Villanueva V, Peltola J, BenMenachem E. Eslicarbazepine acetate: its effectiveness as adjunctive therapy in clinical trials and open studies. J Neurol. 2017 Mar;264(3):421-431. doi: 10.1007/s00415-016-8338-2. Epub 2017 Jan 18. PMID: 28101651; PMCID: PMC5336540.

3. Verrotti A, Loiacono G, Rossi A, Zaccara G. Eslicarbazepine acetate: an update on efficacy and safety in epilepsy. Epilepsy Res. 2014 Jan;108(1):1-10. doi: 10.1016/j.eplepsyres.2013.10.005. Epub 2013 Oct 23. PMID: 24225327.

4. Bialer M, Soares-da-Silva P. Pharmacokinetics and drug interactions of eslicarbazepine acetate. Epilepsia. 2012 Jun;53(6):935-46. doi: 10.1111/j.15281167.2012.03519.x. Epub 2012 May 21. PMID: 22612290.

5. Alcántara Montero A, Sánchez Carnerero CI. Eslicarbazepine acetate for neuropathic pain, headache, and cranial neuralgia: Evidence and experience. Neurologia (Engl Ed). 2019 Jul-Aug;34(6):386-395. English, Spanish. doi: 10.1016/j.nrl.2016.11.009. Epub 2017 Feb 16. PMID: 28215909. 
6. Galiana GL, Gauthier AC, Mattson RH. Eslicarbazepine Acetate: A New Improvement on a Classic Drug Family for the Treatment of Partial-Onset Seizures. Drugs R D. 2017 Sep;17(3):329-339. doi: 10.1007/s40268-017-0197-5. PMID: 28741150 ; PMCID: PMC5629137.

7. Lattanzi S, Brigo F, Cagnetti C, Verrotti A, Zaccara G, Silvestrini M. Eslicarbazepine acetate in the treatment of adults with partial-onset epilepsy: an evidence-based review of efficacy, safety and place in therapy. Core Evid. 2018 Mar 8;13:21-31. doi: 10.2147/CE.S142858. PMID: 29563858; PMCID: PMC5846310.

8. Trinka E, Rocamora R, Chaves J, Moreira J, Ikedo F, Soares-da-Silva P; BIA-2093311/EXT Investigators Study Group. Long-term efficacy and safety of eslicarbazepine acetate monotherapy for adults with newly diagnosed focal epilepsy: An open-label extension study. Epilepsia. 2020 Oct;61(10):2129-2141. doi: 10.1111/epi.16666. Epub 2020 Sep 17. PMID: 32944934; PMCID: PMC7693183.

9. Pazdera L, Sperling MR, Harvey JH, Sam MC, Strom LA, Blum D, Grinnell T, Cheng H. Efficacy and safety of eslicarbazepine acetate monotherapy in patients converting from carbamazepine. Epilepsia. 2018 Mar;59(3):704-714. doi: 10.1111/epi.14014. Epub 2018 Feb 16. PMID: 29450890.

10. Sperling MR, French J, Jacobson MP, Pazdera L, Gough M, Cheng H, Grinnell T, Blum D; Study 045 and 046 Investigators. Conversion to eslicarbazepine acetate monotherapy: A pooled analysis of 2 phase III studies. Neurology. 2016 Mar 22;86(12):1095-102. doi: 10.1212/WNL.0000000000002497. Epub 2016 Feb 24. PMID: 26911639; PMCID: PMC4826334.

11. Sperling MR, Abou-Khalil B, Harvey J, Rogin JB, Biraben A, Galimberti CA, Kowacs PA, Hong SB, Cheng H, Blum D, Nunes T, Soares-da-Silva P; 304 Study Team. Eslicarbazepine acetate as adjunctive therapy in patients with uncontrolled partial-onset seizures: Results of a phase III, double-blind, randomized, placebocontrolled trial. Epilepsia. 2015 Feb;56(2):244-53. doi: 10.1111/epi.12894. Epub 2014 Dec 22. PMID: 25528898; PMCID: PMC4354260

12. Costa R, Steinhoff B, Gama H, Ikedo F, Rocha JF, Soares-da-Silva P. Safety, Tolerability and Efficacy of Eslicarbazepine Acetate as Adjunctive Therapy in Patients Aged $\geq 65$ Years with Focal Seizures. Drugs Aging. 2018 Dec;35(12):11091117. doi: 10.1007/s40266-018-0602-y. PMID: 30387043; PMCID: PMC6267536.

13. Hufnagel A, Ben-Menachem E, Gabbai AA, Falcão A, Almeida L, Soares-da-Silva P. Long-term safety and efficacy of eslicarbazepine acetate as adjunctive therapy in the treatment of partial-onset seizures in adults with epilepsy: results of a 1-year openlabel extension study. Epilepsy Res. 2013 Feb;103(2-3):262-9. doi: 10.1016/j.eplepsyres.2012.07.014. Epub 2012 Aug 4. PMID: 22871333.

14. Abraira L, Sanabria A, Ortega G, Quintana M, Santamarina E, Salas-Puig J, Toledo M. Cognicion social y funciones cognitivas en pacientes con epilepsia tratados con acetato de eslicarbacepina [Social cognition and cognitive functions in patients with epilepsy treated with eslicarbazepine acetate]. Rev Neurol. 2018 Jun 1;66(11):361-367. Spanish. PMID: 29790568.

15. Vaz-Da-Silva M, Nunes T, Almeida L, Gutierrez MJ, Litwin JS, Soares-Da-Silva P. Evaluation of Eslicarbazepine acetate on cardiac repolarization in a thorough QT/QTc 
study. J Clin Pharmacol. 2012 Feb;52(2):222-33. doi: 10.1177/0091270010391789. PMID: 21415284.

16. Mintzer S, Wechsler RT, Rogin JB, Gidal BE, Schwab M, Ben-Menachem E, Carreño M, da Silva PS, Moreira J, Li Y, Blum D, Grinnell T. Effects of adjunctive eslicarbazepine acetate on serum lipids in patients with partial-onset seizures: Impact of concomitant statins and enzyme-inducing antiepileptic drugs. Epilepsy Res. 2018 Mar;141:83-89. doi: 10.1016/j.eplepsyres.2018.02.001. Epub 2018 Feb 9. PMID: 29499473.

17. Falcão A, Pinto R, Nunes T, Soares-da-Silva P. Effect of repeated administration of eslicarbazepine acetate on the pharmacokinetics of simvastatin in healthy subjects. Epilepsy Res. 2013 Sep;106(1-2):244-9. doi: 10.1016/j.eplepsyres.2013.04.009. Epub 2013 May 30. PMID: 23726291.

18. Gidal BE, Mintzer S, Schwab M, Schutz R, Kharidia J, Blum D, Grinnell T, Sunkaraneni S. Evidence for a pharmacokinetic interaction between eslicarbazepine and rosuvastatin: Potential effects on xenobiotic transporters. Epilepsy Res. 2017 Sep;135:64-70. doi: 10.1016/j.eplepsyres.2017.05.005. Epub 2017 May 18. PMID: 28624574 . 